\title{
Patients receiving maintenance dialysis have more severe functionally significant skeletal muscle wasting than patients with dialysis-independent chronic kidney disease
}

\author{
Christopher W. McIntyre ${ }^{1,2}$, Nicholas M. Selby ${ }^{1}$, Mhairi Sigrist ${ }^{1}$, Lyndsay E. Pearce ${ }^{1}$, \\ Thomas H. Mercer ${ }^{3}$ and Patrick F. Naish ${ }^{4}$ \\ ${ }^{1}$ Department of Renal Medicine, Derby City General Hospital, Derby, ${ }^{2}$ Centre for Integrated Systems Biology and \\ Medicine, University of Nottingham, ${ }^{3}$ School of Sport, Health and Exercise Sciences, University of Wales, \\ Bangor and ${ }^{4}$ Department of Nephrology, North Staffordshire Hospital Trust, Stoke-on-Trent, UK
}

\begin{abstract}
Background. Chronic renal replacement therapy patients exhibit reduction in skeletal muscle function as a result of a combination of metabolic effects and muscle fibre size reduction. The aim of this study was to compare muscle mass with function in patients with chronic kidney disease (CKD) at stages 4 and 5 on haemodialysis (HD) and peritoneal dialysis (PD), and investigate the associations of muscle wasting in a cross-sectional cohort.

Methods. We studied 134 patients (60 HD, 28 PD and 46 CKD 4). The three groups were well matched for age, sex, diabetes and dialysis vintage. Cross-sectional area (CSA) of muscle and fat was measured from a standardized multi-slice CT scan of a $6 \mathrm{~cm}$ long section of thigh. CSA of soft tissue was taken from appropriate fat and muscle densities. Functional assessment was by the sit-to-stand 60 test, assessing both the number of sit-to-stands possible under controlled conditions in $60 \mathrm{~s}$ (STS 60), and the time taken to perform five sit-to-stand movements (STS 5). Data were collected on a wide range of potential determinants of muscle CSA.

Results. There were no significant differences in haemoglobin between males or females or between any of the groups studied. Serum phosphate and calcium-phosphate product were higher in HD patients as compared to CKD4 patients, but there were no differences in these variables when comparing PD patients with either CKD4 or HD patients. Muscle CSA correlated well with objective functional assessments in males (STS $60 R=0.52, P<0.0001$ ) and females $(R=0.41, P=0.004)$, and STS performance

Correspondence and offprint requests to: Dr C McIntyre, Department of Renal Medicine, Derby City General Hospital, Uttoxeter Rd, Derby, DE22 3NE, UK.

Email: Chris.McIntyre@derbyhospitals.nhs.uk
\end{abstract}

was reduced in dialysed patients as compared with CKD 4. Univariate analysis demonstrated that muscle CSA was associated with serum albumin concentration $(R=0.49, P<0.0001)$, age $(R=-0.35, P=0.005)$ and C-reactive protein $(R=-0.34, P=0.004)$. Creatinine clearance, dialysis adequacy, dialysis vintage and timeaveraged serum bicarbonate, calcium and phosphate concentrations were not correlated with muscle CSA. Conclusion. In conclusion, patients with dialysistreated CKD 5 exhibited more functionally significant muscle wasting than patients with CKD 4. This may be amenable to modification with targeted exercise or amelioration of factors associated with observed differences in muscle mass.

Keywords: chronic kidney disease; computed tomography; haemodialysis; peritoneal dialysis; skeletal muscle; wasting

\section{Introduction}

Chronic kidney disease (CKD) is associated with both muscle wasting $[1,2]$ and reduced functional capacity [3]. Reduction in physical conditioning is associated with both reduced quality of life and increased mortality in patients receiving dialysis. [4].

Reduced muscle strength and endurance in dialysis patients is multifactorial and incompletely elucidated. Although the ability to generate force per muscle unit (myopathy) [5] and reduction in CNS-activation of normal motor units (central activation failure) appear to be important, reduction in muscle mass (atrophy) has been demonstrated in a variety of studies [6].

The uraemic milieu itself appears to predispose to reduced exercise capacity and muscle atrophy. Long-term 
inflammatory exposure [7], poor nutritional status [8], elevated circulating cytokine levels and reductions in insulin-like growth factors, both circulating and within skeletal muscle [9], have all been reported to be associated with reductions in functional muscle mass.

Muscle mass can be assessed using both invasive and non-invasive methods. Direct quantification by measurement of muscle cross-sectional area (CSA) using both magnetic resonance imaging [1] or computed tomography (CT) [2] have been utilized. Muscle CSA has been reported as an accurate and practical method to detect functionally significant muscle wasting in dialysis patients and correlates closely with direct muscle mass measurements from cadavers [10]. Muscle biopsy studies have revealed abnormal skeletal muscle architecture in patients with CKD. This is particularly marked in those receiving dialysis. Most studies have been confined to haemodialysis (HD) patients. Atrophy of type II fibres is most prominent [5,11], with abnormal capillarization [12]. This appears to be a particular feature in HD patients. Controversy still exists on the relative importance of these various factors and the overall impact of the uraemic state itself on skeletal muscle mass and function in CKD patients.

The aim of this study was to examine skeletal muscle mass and function in a cross-sectional study of a single cohort of CKD patients. For the first time this would allow comparison of CKD 4 patients with advance uraemia and those with CKD 5 receiving either HD or peritoneal dialysis (PD).

\section{Methods}

\section{Patients}

We studied 134 subjects (60 receiving HD, $28 \mathrm{PD}$ and 46 CKD 4). All subjects were recruited from Derby City General Hospital. All HD, PD and CKD 4 patients were eligible to enter the study unless they had been previously transplanted, or had an amputated limb. All eligible patients registered under the care of the renal consultants were approached to take part in this study (total population of $158 \mathrm{HD}, 71 \mathrm{PD}$ and 62 CKD 4 in May 2003, at the start of recruitment). Their characteristics are summarized in Table 1 . There were no significant differences in age, sex or prevalence of diabetes between the three groups, and no difference in dialysis vintage between patients on PD or HD. CKD 4 patients were defined as having at least two creatinine clearance measurements made, with values between $15-30 \mathrm{ml} / \mathrm{min}$. Retrospective application of the 4-point modification of diet in renal disease (MDRD) formula to calculate estimated glomerular filtration rate (GFR) confirmed that all patients had been in stage 4 for at least 6 months prior to recruitment. Dialysis modality was a matter of free patient choice, unless an overarching medical imperative existed that prevented the use of PD. No patients were included who had changed dialysis modality.

PD patients were all treated with bicarbonate/lactate-mixbuffered PD fluids (Physioneal). A total of nine out of 28 PD patients were on automated PD and the others on continuous ambulatory peritoneal dialysis (3-5 exchanges/day). All patients had been established on PD for at least 6 months.
HD patients had been established on HD for at least 6 months. All patients were receiving three sessions of at least $4 \mathrm{~h}$ duration per week (maximum $5 \mathrm{~h}$ per session). Dialysis was performed using Hospal Integra (Mirandola, Italy) monitors and low-flux polysulphone dialysers $\left(1.5-2.0 \mathrm{~m}^{2}\right.$, LOPS $15-20^{\circledR}$, Braun Medical Ltd, Sheffield, UK). All patients were dialysed using a $1.25 \mathrm{mEq} / 1$ calcium and $134 \mathrm{mmol} / 1$ sodium-containing dialysate. All patients received bicarbonate-based HD. Patients were all dialysed at the main dialysis centre at Derby City Hospital.

Patients were excluded if they had ongoing conditions likely to increase muscle catabolism, including HIV infection, active sepsis or known malignancy. Patients were receiving standard dietary advice on their intake, no formal assessment of their nutritional status was undertaken. Comorbidities and current medications were recorded from review of the patients' notes. Daily sodium intake was limited to $100 \mathrm{mmol}$ and daily protein intake was set at $1.2 \mathrm{~g} / \mathrm{kg} / \mathrm{day}$, this advice had been followed for at least 1 year prior to the study. A total of $95 \%$ of the patients on HD and $82 \%$ of patients were receiving epoeitin therapy. Appropriate ethical approval had been granted by the South Derbyshire Local Research Ethics Committee, and all patients provided written consent.

\section{Data collection}

Date were recorded for all patients on age, sex, comorbidities, medication, BMI, height, time-averaged (for previous 6 months) haemoglobin, serum-phosphate, serum-corrected calcium, albumin (bromocresol purple method) and serum bicarbonate concentration. C-reactive protein (CRP) was measured at the last routine monthly blood-taking, prior to functional assessment and measurement of muscle CSA. In addition, dialysis patients had their last dialysis adequacies recorded (all within 1 month of assessment) as well as dialysis details and time on renal replacement therapy.

\section{Imaging}

All studies were performed using GE Medical Systems lightspeed $16^{\circledR}$ multi-slice spiral CT scanner. Images were acquired when the patient was supine, no contrast was used. A standardized section through the thigh, $20 \mathrm{~cm}$ above the tibial plateau, $6 \mathrm{~cm}$ in length was imaged in $2.5 \mathrm{~mm}$ slices, care was taken to ensure that none of the slices overlapped. Actual scan time was found to be under a minute. A medical physicist calculated radiation exposure. The dose of radiation given for the overall scout picture was found to be $8.175 \mathrm{mgy}$. The dose of the radiation in order to image the specific section of the inner thigh where this section of the superficial femoral artery is located was found to be $108 \mathrm{mgy}$. This equates to a smaller dose of radiation than a chest X-ray, which has an average radiation dose of $120 \mathrm{mgy}$.

The scans were scored by two people, blinded for both dialysis modality and patient identity. Scoring was undertaken using GE Medical Systems ${ }^{\circledR}$ Advantage Workstation software. Muscle CSA $\left(\mathrm{mm}^{2}\right)$ was measured after an appropriate area of interest was drawn around the images and windowing for the appropriate densities (fat and noncontractile connective tissue $-200-0 \mathrm{HU}$, muscle $0-200 \mathrm{HU}$ ). Muscle CSA was measured of the top, middle and bottom slices of this $6 \mathrm{~cm}$ cylinder of thigh, and the mean of these three values was then adjusted for height. This was an attempt 
Table 1. Descriptive summary of all patient demographics, biochemical parameters and relevant prescribed medications included in the study, by modality. Results are expressed as mean \pm SD or number of observations (percentage of total). Results are analysed using oneway ANOVA, with Tukey post-test for normally distributed data or Chi-square tests for non-parametric data

\begin{tabular}{|c|c|c|c|c|}
\hline & CKD $(n=46)$ & $\mathrm{PD}(n=28)$ & $\mathrm{HD}(n=60)$ & Significant $P$-value \\
\hline Age (years) & $60 \pm 14$ & $61 \pm 14$ & $60 \pm 15$ & NS \\
\hline Male gender & $26(56 \%)$ & $17(60 \%)$ & $42(70 \%)$ & NS \\
\hline Diabetes mellitus & $10(21 \%)$ & $8(29 \%)$ & $16(27 \%)$ & NS \\
\hline Smokers & $6(13 \%)$ & $4(14 \%)$ & $6(10 \%)$ & NS \\
\hline Dialysis vintage (months) & $\mathrm{n} / \mathrm{a}$ & $34 \pm 23$ & $36 \pm 25$ & NS \\
\hline Body mass index $\left(\mathrm{kg} / \mathrm{m}^{2}\right)$ & $25 \pm 9$ & $25 \pm 11$ & $23 \pm 9$ & NS \\
\hline Creatinine clearance $(\mathrm{ml} / \mathrm{min})$ & $19 \pm 6$ & $8 \pm 3$ & $2 \pm 5$ & 0.0001 \\
\hline Dialysis adequacy $(\mathrm{Kt} / \mathrm{V})^{\mathrm{a}}$ & - & $2.5 \pm 0.5$ & $1.2 \pm 0.2$ & - \\
\hline Previous CV comorbidities ${ }^{\mathrm{b}}$ & $11(23 \%)$ & $12(42 \%)$ & $12(20 \%)$ & NS \\
\hline Total cholesterol (mmol/l) & $5.1 \pm 0.9$ & $4.5 \pm 1.0$ & $4.3 \pm 1.0$ & $\begin{array}{l}0.002 \\
<0.05^{*} \\
<0.001^{* *} \\
\text { NS*** }\end{array}$ \\
\hline HDL cholesterol $(\mathrm{mmol} / \mathrm{l})$ & $1.3 \pm 0.3$ & $1.3 \pm 0.3$ & $1.3 \pm 0.3$ & NS \\
\hline LDL cholesterol $(\mathrm{mmol} / \mathrm{l})$ & $2.7 \pm 0.9$ & $2.6 \pm 0.9$ & $2 \pm 0.7$ & $\begin{array}{l}0.0003 \\
\text { NS* } \\
<0.001 * * \\
<0.05^{* * *}\end{array}$ \\
\hline Serum phosphate $(\mathrm{mmol} / \mathrm{l})$ & $1.49 \pm 0.3$ & $1.59 \pm 0.3$ & $1.70 \pm 0.4$ & $\begin{array}{l}0.01 \\
\mathrm{NS}^{*}, * * * \\
<0.01^{* *}\end{array}$ \\
\hline Serum albumin corrected calcium $(\mathrm{mmol} / \mathrm{l})$ & $2.38 \pm 0.1$ & $2.51 \pm 0.1$ & $2.46 \pm 0.1$ & $\begin{array}{l}<0.001 \\
<0.001^{*} \\
<0.001^{* *} \\
\mathrm{NS}^{* * *}\end{array}$ \\
\hline Calcium X phosphate product $\left(\mathrm{mmol}^{2} / 1^{2}\right)$ & $3.5 \pm 0.6$ & $4.0 \pm 0.7$ & $4.2 \pm 1.1$ & $\begin{array}{l}0.001 \\
\mathrm{NS}^{*}, * * * \\
<0.01^{* *}\end{array}$ \\
\hline PTH (pg/ml) & $196 \pm 105$ & $293 \pm 267$ & $287 \pm 278$ & NS \\
\hline Albumin $(\mathrm{g} / \mathrm{l})$ & $34 \pm 2.8$ & $27 \pm 3.7$ & $33 \pm 4.2$ & $P<0.001$ \\
\hline C-reactive protein $(\mathrm{mg} / \mathrm{l})$ & $10 \pm 16$ & $15 \pm 40$ & $21 \pm 29$ & NS \\
\hline Use of Vitamin D & $17(37 \%)$ & $17(61 \%)$ & $33(55 \%)$ & NS \\
\hline Lipid lowering therapy & $15(33 \%)$ & $13(46 \%)$ & $18(30 \%)$ & NS \\
\hline Use of non-calcium-based phosphate binders & $1(2 \%)$ & $16(57 \%)$ & $26(43 \%)$ & $<0.001$ \\
\hline Use of calcium-based phosphate binders & $17(37 \%)$ & $13(46 \%)$ & $37(61 \%)$ & 0.04 \\
\hline Calcium channel blockers & $23(50 \%)$ & $11(39 \%)$ & $13(21 \%)$ & NS \\
\hline Use of ACE inhibitors & $17(37 \%)$ & $11(89 \%)$ & $11(18 \%)$ & NS \\
\hline Use of $\beta$ blockers & $20(43 \%)$ & $3(10 \%)$ & $15(38 \%)$ & $P=0.01$ \\
\hline
\end{tabular}

NS, non-significant.

*Comparison between CKD 4 and PD; **Comparison between CKD 4 and HD; ***Comparison between PD and HD.

${ }^{\mathrm{a}} \mathrm{Kt} / \mathrm{V}$ in PD is 'Weekly' and in HD is 'per single session'.

${ }^{\mathrm{b}} \mathrm{CV}$ comorbidities are defined as any previous description of ischaemic heart disease, heart failure, cerebral vascular disease or peripheral vascular disease recorded in the patients' medical notes.

to capture a comparable distribution of thigh muscle and fat content, allowing for the differing morphologies of the thigh between patients at this level.

\section{Functional muscle assessment}

Simple objective tests of overall physical function were undertaken. The tests involve rising, with arms folded across the chest, from the sitting position from a chair seat of standard height $(\sim 46 \mathrm{~cm})$ and returning to the sitting position. The movement was explained and demonstrated and the patient was habituated to the procedure. Patients were timed whilst performing five repetitions of the sit-tostand test as quickly as they could (STS 5; a surrogate measure of muscle power), and also the maximum number of sit-to-stand tests that could be performed in $60 \mathrm{~s}$ was also recorded (STS 60; a surrogate measure of muscle endurance).
This type of assessment of physical performance has been widely used in the study of CKD patients [13], and well validated against more detailed assessments [14].

\section{Statistical analysis}

Group data are represented as mean \pm SD $(95 \%$ confidence intervals) unless otherwise stated. All data were tested for normality. Comparisons between groups were made initially by one-way ANOVA and post-test Bonnferroni's multiplecomparison test, and subsequently using unpaired $t$-test where appropriate. Muscle CSA between CKD patient groups is displayed as box and whisker plots to best demonstrate the spread of data visually.

Univariate regression analysis was used to assess the impact of potential determinants on muscle mass. CRP levels were not normally distributed and were analysed log transformed. Linear regression analysis was also performed 
to study the relationship between muscle CSA and functional performance. All analyses were performed using Prism v3.0 software package.

\section{Results}

Muscle mass, as assessed by the measurement of muscle CSA corrected for linear height, was significantly lower in patients receiving dialysis (by about 9\%), as compared with patients with CKD 4 (Table 2). This is despite the patients being well matched for age, sex, prevalence of diabetes and dialysis vintage (for those on HD or PD) (Table 1). On average, males exhibited around $8 \%$ greater muscle mass than females. There were no significant differences in muscle CSA between patients receiving $\mathrm{HD}$ or PD (Figure 1). These differences between CKD 4 patients and those receiving dialysis were also reflected in higher levels of physical performance in the CKD 4 patients (Table 3). There was a non-statistically significant trend for an increased thigh CSA for fat (uncorrected for height) in PD patients as compared with CKD 4 and HD patients $\left(10220 \pm 1004 \mathrm{~mm}^{2}\right.$, $9391 \pm 734 \mathrm{~mm}^{2}$ and $8858 \pm \mathrm{mm}^{2}$, respectively, $\left.P=0.06\right)$.

Reductions in muscle CSA appeared to be functionally significant. Muscle CSA was positively correlated with STS 60 and negatively correlated with STS 5 assessments of overall physical condition in both males and females (all $P<0.001$, Figure 2). These relationships were also present when the data were analysed by individual dialysis modality or in CKD 4 patients alone.

When considering all patients together univariate analysis demonstrated that only CRP, age and serum albumin were

Table 2. Univariate analysis of correlation of various factors with muscle CSA corrected for linear height $(n=134)$

\begin{tabular}{lcc}
\hline & $R$ & $P$ \\
\hline Serum albumin & 0.49 & $<0.0001$ \\
Age & -0.35 & 0.005 \\
Log CRP & -0.34 & 0.004 \\
Creatinine clearance & 0.08 & 0.56 \\
Dialysis adequacy & 0.13 & 0.6 \\
Serum bicarbonate & 0.03 & 0.81 \\
Time on dialysis & 0.14 & 0.39 \\
Serum corrected calcium & 0.1 & 0.51 \\
Serum phosphate & 0.14 & 0.18 \\
\hline
\end{tabular}

significantly correlated with muscle CSA, while creatinine clearance (CKD 4 patients), weekly creatinine clearance (PD patients), delivered Kt/V (HD patients), time-averaged serum bicarbonate, serum calcium and serum phosphate were not correlated (Table 3). There were no significant differences in Haemoglobin between males or females or between any of the groups studied. Serum phosphate and calcium-phosphate product were higher in HD patients as compared to CKD4 patients, but there were no differences in these variables when comparing PD patients with either CKD4 or HD patients. Serum albumin concentration was lower in PD patients (mean albumin $27.1 \pm 3.9 \mathrm{~g} / \mathrm{l}$ ) than CKD 4 patients (mean albumin $34.2 \pm 2.9 \mathrm{~g} / 1, P<0.0001$ ), and HD patients (mean albumin $34.3 \pm 1.3, P<0.0001)$. There was no significant difference between time-averaged serum albumin concentration between CKD 4 patients and HD patients $(P=0.77)$. Mean CRP concentrations were significantly lower in CKD 4 patients $(7.1 \pm 7.2 \mathrm{mg} / \mathrm{l})$ than either HD $(29.1 \pm 57.2 \mathrm{mg} / 1, P=0.03)$ or PD patients $(33.6 \pm 41.7 \mathrm{mg} / 1, P=0.001)$. Mean CRP concentrations were not significantly higher in HD patients compared with PD patients $(P=0.73)$. All sex specific data for each of the three patient groups are summarized in Table 2.

Although the overall level of physical activity between the three groups was not formally assessed, there were no significant differences in the percentage of patients who were unable to perform the STS tests (Table 2).

\section{Discussion}

This cross-sectional study allows the first direct assessment of muscle CSA and functional capacity in CKD 4 patients with those receiving both PD and HD. Functionally significant muscle atrophy is a common feature of uraemia and is more pronounced in patients receiving dialysis than those with CKD 4.

Unsurprisingly, muscle CSA was greater in males rather than females. This difference was maintained in CKD 4 and 5 patients, as was the normal association of age with CSA. This association with age has been reported using a similar methodology to that used in this study, but without reference to other uraemic comparative groups or measures of muscular function [15]. The amount of muscle, as assessed by measured
A

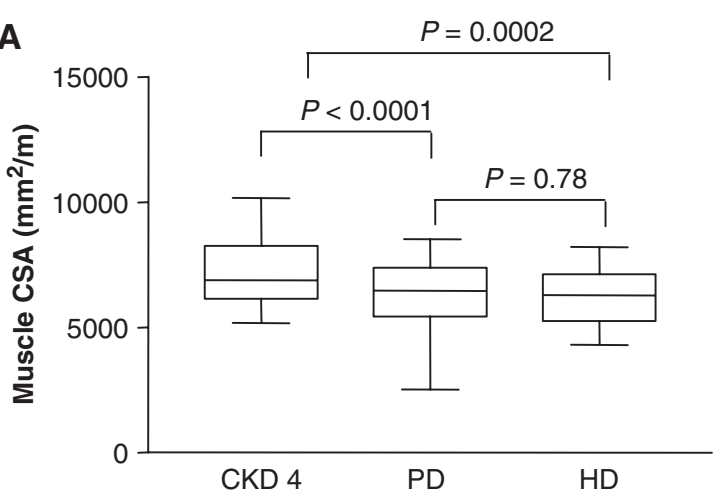

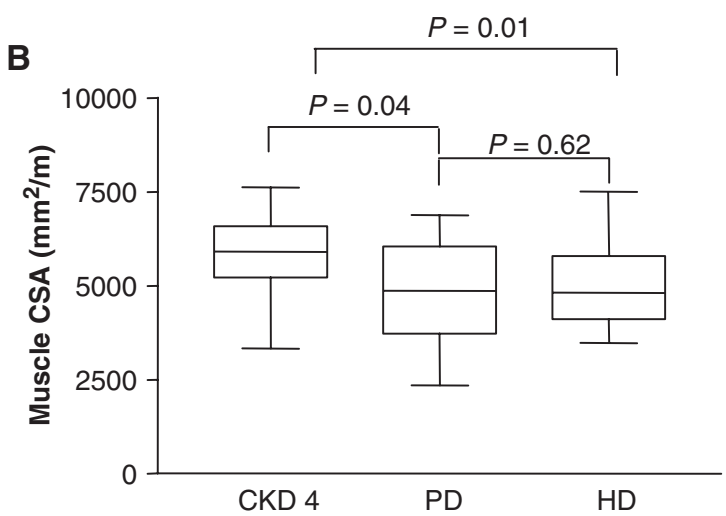

Fig. 1. Box and whisker plots (median, 25-75th quartile and overall data ranges) for height-corrected muscle CSA comparing CKD 4, $\mathrm{PD}$ and HD patients in males (A) and females (B). 
thigh CSA corrected for height, did correlate with objective measures of physical performance. Significant reduction in muscle CSA was associated with significant reduction in functional capacity. There was a trend to increased fat CSA in PD patients as compared with CKD 4 and HD patients, possibly associated with the systemic exposure of an increased carbohydrate load from glucose-based dialysis solutions.

The determinants of muscle atrophy are likely to be multifactorial, and potentially have differing relative effects in patients with CKD 4 as compared with those receiving either $\mathrm{PD}$ or $\mathrm{HD}$. Differences might also exist in the aetiology of wasting between PD and HD patients. This is consistent with the observed differences in capillarization in muscle biopsy studies between dialysis modalities [12]. It would appear from our study that CKD 5 patients do exhibit a similar muscle mass, regardless of dialysis modality, and this is less than in patients who are non-dialysis dependant but with pronounced uraemia. A reduced muscle mass (in tibialis anterior muscle) has already been reported in HD patients in comparison with non-uraemic controls [1]. The net effect of dialysis (irrespective of modality) on both muscle mass and functional capacity appears to be similar. This is the first study that has allowed direct comparison of measured muscle amount between these groups. Other studies relying on indirect methods such as anthropometry tend to underestimate muscle wasting [16].
Atrophy is obviously also a result of disuse. However, histological study of non-locomotor muscles in CKD 5 patients does reveal atrophic change, consistent with direct effects of the uraemic milieu [6]. Although no direct measurements of overall physical activity were made between these patients, the lack of significant difference in the percentage of patients unable to complete the STS assessment, and similar overall levels of performance as measured by that assessment suggests there was little difference. Increased levels of debility in one dialysis modality compared with the other is also unlikely, given the similar demographics, dialysis vintage and essentially free choice of modality that had been offered to patients. $\mathrm{Hb}$ concentration was also similar between the three groups. Anaemia was therefore unlikely to have contributed to differences in functional status.

CKD is associated with reduced muscle protein synthesis as compared with non-uraemic controls [17]. No data are available on whether or not this is proportional to the severity of uraemia. Acidosis has been reported to be important in the control of muscle protein synthesis and catabolism [18]. However, there were no observed differences in serum bicarbonate concentration between CKD 4 or 5 patients, and serum bicarbonate concentration was not correlated with muscle CSA. This may, however, be an important mechanism for muscle wasting if compared with the non-uraemic population, as a significant percentage
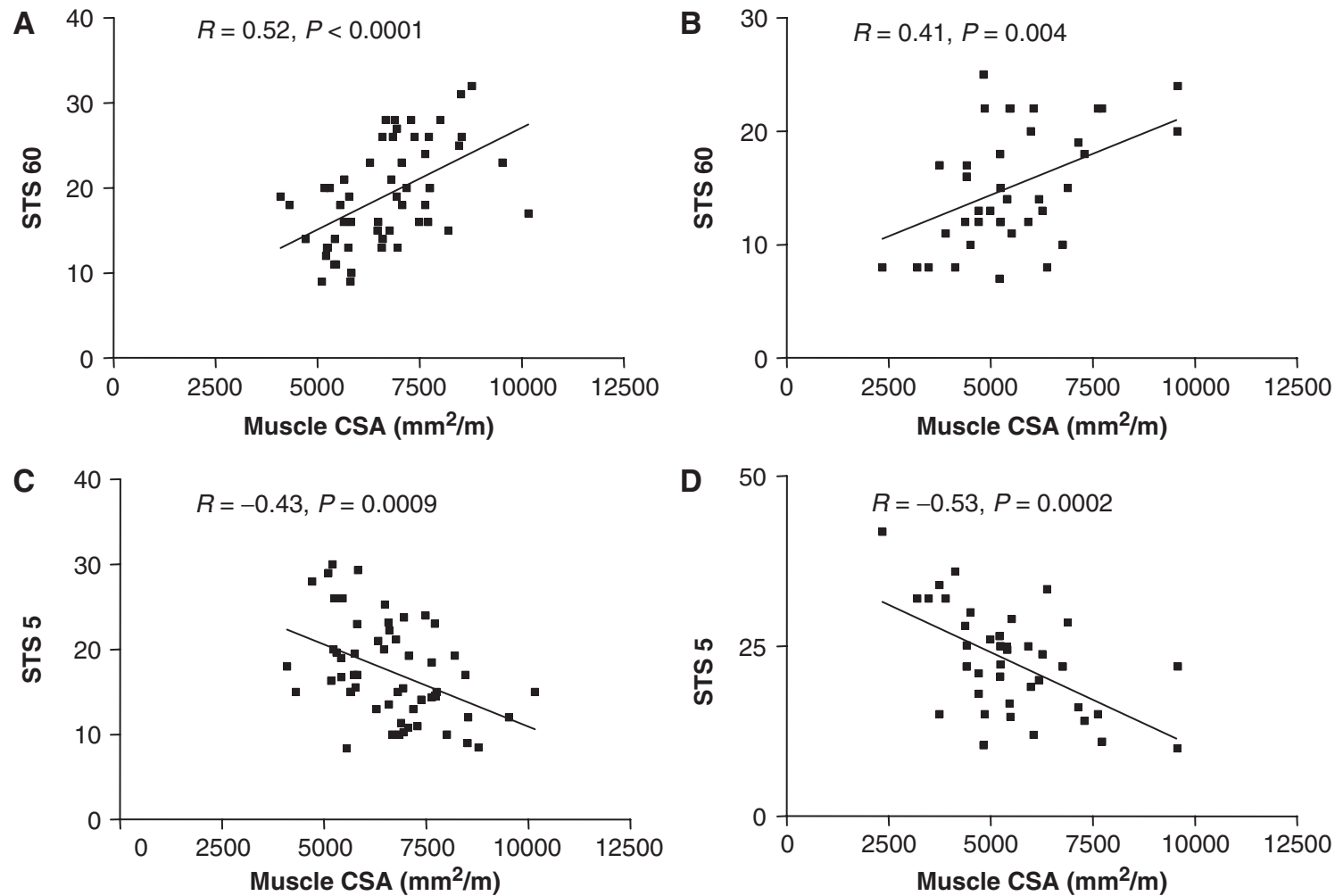

Fig. 2. Linear correlations between height-corrected muscle CSA and functional STS tests in males (A and C) and females (B and D). This demonstrates reduced muscle CSA is associated with a reduced number of STS movements in $60 \mathrm{~s}$ and a longer period of time to complete 5 STS in patients capable of undertaking this assessment. 
of the patients studied did have reduced serum bicarbonate concentrations.

Protein energy malnutrition (PEM) is prevalent in patients receiving dialysis [8] and is associated with muscle wasting [19]. Serum albumin is a widely used marker of nutritional status and was the most strongly correlated factor with reduced muscle CSA. The lower serum albumin concentrations seen in the patients on PD are less indicative of PEM and are largely a result of peritoneal protein losses, particularly in those patients with higher transporter status.

Serum albumin, though is a poor marker of overall nutritional status, may be a marker also of the level of generalized inflammatory stress. Systemic inflammation with suppression of circulating protein synthesis and elevation in acute-phase proteins, is another critical component of the malnutrition/inflammation-complex characteristic of many uraemic patients. It is also associated with reduced muscle mass [20]. The fact that serum CRP concentrations are commonly elevated in both PD and HD patients has been appreciated for a number of years [21], and were elevated in this study as compared with CKD 4 patients. CRP concentrations were negatively associated with muscle CSA. The association, though not particularly strong, may well have reflected the single value of CRP used in the analysis rather than time-averaged values that better reflect previous exposure to chronic inflammation.

This study has a number of limitations, a number of which are inherent in the cross-sectional observation approach, which limit the usefulness of extensive investigation of true determinants of presumed progressive muscle wasting. For instance, the univariate relationship between muscle CSA and STS only explains $16-27 \%$ of the common variance. There are a number of other potential factors that may account for this. The use of stature to 'normalize' muscle CSA may not be ideal, whereas the use of single fascicle length would be ideal, but not possible in this initial investigation. The choice of muscles in the calculation of muscle CSA to include others not used in the main movements studied is also a potential weakness. This approach was chosen as being both relatively simple to apply, and most consistent with published literature on the use of thigh CT assessment of muscle, but could be refined in further study. An approach based on physiological rather than anatomical CSA might also improve the correlations with functional performance.

In conclusion, patients with dialysis-treated CKD 5 exhibited more functionally significant muscle wasting even than CKD 4 patients with significant uraemia. This may be amenable to modification with targeted exercise or amelioration of factors associated with observed differences in muscle mass. Serial assessments with CT offer an excellent opportunity to study the determinants of change in muscle mass in patients established on dialysis or transiting from CKD 4 to 5.

Conflict of interest statement. None declared. 


\section{References}

1. Johansen K, Shubert T, Doyle J et al. Muscle atrophy in patients receiving hemodialysis: effects on muscle strength, muscle quality and physical function. Kidney Int 2003; 63: 291-297

2. Ohkawa S, Odamaki M, Yoneyama $\mathrm{T}$ et al. Standaradised thigh muscle area measured by computed axial tomography as an alternate muscle mass index for nutritional assessment of hemodialysis patients. Am J Clin Nutr 2000; 71: 485-490

3. Beasley C, Smith D, Neale T. Exercise capacity in chronic renal failure patients managed by continuous ambulatory peritoneal dialysis. Aust NZ J Med 1986; 16: 5-10

4. DeOreo P. Hemodialysis patient-assessed functional health status predicts continued survival, hospitalisation and dialysis attendance compliance. Am J Kidney Dis 1997; 30: 204-212

5. Kouidi E, Albani M, Natkis $\mathrm{K}$ et al. The effects of exercise training on muscle atrophy in haemodialysis patients. Nephrol Dialysis Transplant 1998; 13: 685-699

6. Sakkas G, Ball D, Mercer T et al. Atrophy of non-locomotor muscle in patients with end-stage renal failure. Nephrol Dialysis Transplant 2003; 18: 2074-2081

7. Kaizu Y, Ohkawa S, Odamaki M et al. Association between inflammatory mediators and muscle mass in long-term hemodialysis patients. Am J Kidney Dis 2003; 42: 295-302

8. Bergstrom J. Nutrition and mortality in hemodialysis. $J \mathrm{Am}$ Soc Nephrol 1995; 6: 1329-1341

9. Macdonald J, Phanish M, Marcora S et al. Muscle insulin-like growth factor status, body composition and functional capacity in hemodialysis patients. J Ren Nutr 2004; 14: 248-252

10. Mitsiopolous N, Baumgartner R, Heymsfield $\mathrm{S}$ et al. Cadaver validation of skeletal muscle mass measurement by magnetic resonance imaging and computarized tomography. $J$ Appl Physiol 1998; 85: 115-122
11. Diesel W, Knight B, Noakes T et al. Morphologic features of myopathy associated with chronic renal failure. Am J Kid Dis 1993; 22: 677-684

12. Sakkas G, Ball D, Sargeant A et al. Skeletal muscle morphology and capillarization of renal failure patients receiving different dialysis therapies. Clin Sci (Lon) 2004; 107: 617-623

13. Blake C, O'Meara Y. Subjective and objective physical limitations in high-functioning renal dialysis patients. Nephrol Dialysis Transplant 2004; 19: 3124-3129

14. Guralnik J, Ferruci L, Pieper C et al. Lower extremity function and subsequent disability: consistency across studies, predictive models and value of gait speed alone compared with short physical performance battery. J Gerontol A Biol Sci Med 2000; 55: M221-M231

15. Ohkawa S,Odamaki M, Ikegaya $\mathrm{N}$ et al. Association of age with muscle mass, fat mass and fat distribution in non-diabetic haemodialysis patients. Nephrol Dialysis Transplant 2005; 20: 945-951

16. Woodrow G, Oldroyd D, Turney J et al. Measurement of body composition in chronic renal failure: comparison of skinfold anthropometry and bioelectrical impedance with dual energy X-ray absorpiometry. Eur J Clin Nutr 1996; 50: 295-301

17. Adey D, Kumar R, McCarthy $\mathbf{J}$ et al. Reduced synthesis of muscle proteins in chronic renal failure. Am $J$ Endocrinol Metab 2000; 278: 219-225

18. Caso G, Garlick P. Control of muscle protein kinetics by acidbase balance. Curr Opin Clin Nutr Metab Care 2005; 8: 73-76

19. Young G, Kopple J, Lindholm B et al. Nutritional assessment of continuous ambulatory peritoneal dialysis patients: an international study. Am J Kidney Dis 1991; 17: 462-471

20. Kaysen G, Eiserich J. Characteristics and effects of inflammation in end-stage renal disease. Semin Dial 2003; 16: 438-446

21. McIntyre C, Harper L, Mcdougal IC et al. Serum C-reactive protein as a marker for infection and inflammation in regular dialysis patients. Clinical Nephrology 1997; 46: 371-374

Received for publication: 20.9.05

Accepted in revised form: 3.2 .06 